\title{
Efectos de la transformación de fases con respecto a la orientación y velocidad en el nanocable de cobre deformados por tracción
}

\author{
Effects of phase transformation with respect to the orientation and velocity in \\ the traction-deformed copper nanowire
}

\author{
Víctor Augusto Vega Guillén ${ }^{1}$, Justo Rojas-Tapia ${ }^{2}$ \\ Recibido: Enero 2020 - Aprobado: Junio 2020 - Publicado: Junio 2020
}

\begin{abstract}
RESUMEN
Los NCs tienen muchos comportamientos únicos que no se ven en los materiales a nivel volumen. El objetivo del estudio es conocer los efectos de la trasformación de fases con respecto a la orientación y velocidad en el nanocable de cobre deformados por tracción. Debido la necesidad de comprender mejor los mecanismos de deformación mediante simulaciones y el método de dinámica molecular con los potenciales del tipo EAM evaluamos la transformación de fases en la correlación directa entre la curva tensión-deformación, y el porcentaje de la abundancia de pares. En la región elástica en un rango de $60 \%$ a $80 \%$ de átomos se encuentran formando estructuras $\mathrm{FCC}$, unos $10 \%$ a $20 \%$ la estructura $\mathrm{HCp}$, la formación de la estructura BCC es mínima y el porcentaje de otras formaciones están en un rango de $20 \%$ a $40 \%$, afectadas por la velocidad y orientación como resultado el mayor módulo de elasticidad es de $100 \mathrm{GPa}$ y límite de fluencia de $9.0 \mathrm{GPa}$ con una orientación de $\langle 111\rangle$ este fenómeno único permite el diseño de nanosistemas.
\end{abstract}

Palabras claves: Cables de metal nano; cables de cobre nano; propiedades de cables nano; transformación de fase; efecto de superficie.

\begin{abstract}
NCs have many unique behaviors that are not seen in volume level materials. The objective of the study is to know the effects of the transformation of phases with respect to the orientation and speed in the copper nanowire deformed by traction. Due to the need to better understand the deformation mechanisms through simulations and the molecular dynamics method with the potentials of the EAM type, we evaluate the phase transformation in the direct correlation between the stress-strain curve, and the percentage of the abundance of pairs. In the elastic region in a range of $60 \%$ to $80 \%$ of atoms they are forming FCC structures, about $10 \%$ to $20 \%$ the HCp structure, the formation of the BCC structure is minimal and the percentage of other formations are in a range of $20 \%$ to $40 \%$, affected by speed and orientation as a result, the greatest modulus of elasticity is $100 \mathrm{GPa}$ and yield strength of $9.0 \mathrm{GPa}$ with an orientation of $\langle 111\rangle$ this unique phenomenon allows the design of nanosystems.
\end{abstract}

Keywords: Nano metal cables; nano copper cables; nano cables properties; phase transformation; surface effect.

\footnotetext{
${ }^{1}$ Docente de la Escuela Profesional de Ingeniería Metalúrgica de la Universidad Nacional Mayor de San Marcos. Lima, Perú. E-mail: vvegag@unmsm.edu.pe ${ }^{2}$ Docente de la Facultad de Ciencias Físicas de la Universidad Nacional Mayor de San Marcos. Lima, Perú. E-mail: jrojast@unmsm.edu.pe
} 


\section{INTRODUCCIÓN}

El estudio y el conocimiento de las propiedades mecánicas en los nanocables son de vital importancia para su aplicación en la satisfacción de las necesidades humanas y de la industria. La primera estructura en la que se observa la transformación estructural inducida por la superficie fue la FCC en nanocables de Au por (Diao et al., 2003). La energía superficial y la tensión superficial en los materiales nano estructurados tienen propiedades únicas y han estimulado el desarrollo de los nanomateriales. La gran relación superficie a volumen (Ma et al., 2005), (Liang \& Zhou, 2005) es una de las características fundamentales de los nanomateriales. Los átomos que están en la superficie aumentan la energía superficial y la simetría atómica rota en la superficie induce a la tensión superficial (Ibach, 1997), (Cammarata, 1994). La energía superficial y la tensión superficial son de hecho los parámetros termodinámicos dominantes que rigen la estabilidad estructural de los nanos materiales. La energía de la superficie y la tensión de la superficie generalmente dependen de la orientación y la anisotropía. Aunque es difícil de medir estos dos parámetros experimentalmente, han sido predichos por métodos teóricos como el método del átomo incrustado (EAM) (Gumbsch \& Daw, 1991), método de átomo incorporado modificado (MEAM) (Diao et al., 2004) (Gall et al., 2005), (Wan et al., 1999) método de átomo incrustado en la superficie (SEAM) (Haftel, 1993) y función de densidad teoría (DFT) (Kollár, 2003). A pesar de que hay diferencias claras entre los resultados obtenidos de diferentes enfoques teóricos, hay una tendencia constante y es que los planos $\left\{\begin{array}{lll}1 & 1 & 1\end{array}\right\}$ tienen la energía más baja debido a que están muy juntas en su configuración atómica en metales fcc (Ma \& Xu, 2006), (Emundts et al., 2002). En superficies con orientación $\left(\begin{array}{lll}1 & 0 & 0\end{array}\right)$ de metales fcc como Ir, Pt y Au, la capa superficial con un espesor de varias capas atómicas se contrae y se reconstruye en una red hexagonal muy compacta (hcp). La energía de la tensión se produciría debido al desajuste de la red entre los reorientados planos en la dirección $\left(\begin{array}{lll}1 & 1 & 1\end{array}\right)$ de la capa de superficie y la dirección del plano $\left(\begin{array}{lll}0 & 0 & 1\end{array}\right)$ del substrato, impidiendo consecuentemente la transición estructural. Por lo tanto, los efectos de superficie son producto de la velocidad y la orientación factores dominantes que afectan la estructura de los nanocables e incluso puede inducir una transición estructural completa. Con la base de estos descubrimientos, se tiene otras propiedades interesantes de los materiales, como la superficie inducida reorientación del enrejado, transformación de fase martensítica (MT), comportamiento pseudoelástico y efectos de memoria de forma, han sido estudiados y observados desde estructuras fcc (Diao et al., 2003), (Haftel, 1993), (Sutrakar \& Roy Mahapatra, 2011) bcc (Li et al., 2010) y hep (Sutrakar \& Mahapatra, 2008), compuesto de capas de un solo elemento (Ma et al., 2008), (Ma et al., 2008) aleación intermetálica (Saitoh \& Liu, 2009), (Park \& Zimmerman, 2005) e incluso óxido de metal (Kulkarni et al., 2006) o nitruro (Jung et al., 2012) en nanocables compuestos.

\section{MÉTODOS}

La DM es un tipo de simulación molecular computacional que permite obtener el comportamiento o evolución de un sistema (físico, químico, biológico o en los materiales) como función del tiempo. Operacionalmente es un método para generar las trayectorias de los componentes del sistema compuesto de $\mathrm{N}$ partículas por integración numérica directa de las ecuaciones diferenciales de movimiento de Newton (2.1). La tensión para una deformación dada se calcula de acuerdo con el teorema virial que tiene lo siguiente formulario (Park \& Zimmerman, 2005), (Chen \& Chen, 2005):

$$
\sigma_{i j}=\frac{1}{2}\left(\frac{1}{2} \sum_{\alpha=1}^{N} \sum_{\beta \neq \alpha}^{N} U\left(r^{\alpha \beta}\right) \frac{\Delta x_{i}^{\alpha \beta} \Delta x_{j}^{\alpha \beta}}{r^{\alpha \beta}}-\sum_{\alpha=1}^{N} m_{\alpha} x_{i}^{\alpha} x_{j}^{\alpha}\right)
$$

Donde: $\mathrm{V}$ es el volumen del nanocable, $\mathrm{N}$ es el número total de átomos, $x_{i}^{\alpha}$ es el componente i-ésimo de la velocidad de un átomo $\alpha, m_{\alpha}$ es la masa del átomo $\alpha, r^{\alpha \beta}$ es la distancia entre dos átomos $\alpha y \beta, \Delta x^{\alpha \beta}=x_{j}^{\alpha}-x_{j}^{\beta}, U$ es la función de energía potencial, $\mathrm{y} \alpha \beta=\left\|\Delta x_{j}^{\alpha \beta}\right\|$.

Para el análisis de las estructuras atómicas se realizó bajo los conceptos de:

La función de distribución radial (RDF) se utiliza para obtener información acerca de la proporción a la probabilidad de encontrar un átomo en un cascarón esférico a una distancia $r$ de un átomo de referencia.

La técnica de análisis de vecinos comunes o técnica de análisis de pares inducido por Honeycutt y Anderson utilizamos ya que es una herramienta muy útil para el análisis detallado de la configuración de la vecindad de cualquier par de átomos

El parámetro del centro de simetría (PCS) utilizamos con la finalidad de estudiar los defectos estructurales en el sistema como la dislocación parcial y fallas de apilamiento, fue usado el parámetro de centro de simetría (PCS). En este trabajo, se utiliza la propuesta de (Amigo et al., 2014), 2014, se ha asumido que a las dislocaciones parciales corresponden $3.7<\mathrm{Ci}<4.5$, mientras que los valores de 4.5 $<\mathrm{Ci}<6.5$ están relacionados con las fallas de apilamiento. Los átomos superficiales se caracterizan con $\mathrm{Ci}>6.5$.

La primera observación de la transformación estructural inducida por la superficie fue la estructura FCC en nanocables de Au por (Diao et al., 2004). En su estudio en nanocables de $\mathrm{Au}$ en la dirección [ $\left[\begin{array}{lll}1 & 0 & 0\end{array}\right]$ con una sección transversal cuadrada fueron creados con los átomos posicionados de una manera similar al de la red de fcc en volumen con una constante de red de $4.07 \mathrm{~A}^{\circ}$. (Delogu, 2010) ha ilustrado la transformación de fase fcc a bct en nanotubos de Ag bajo condiciones hidrostáticas por simulación con DM utilizando un potencial semiempírico para describir las interacciones entre los átomos. Basado en la tasa de transformación de fase en función de la presión. La caracterización estructural mostró que la transformación de fase de fcc a cuerpo centrado tetragonal (bct) se observa sin el proceso intermedio que involucra la transformación de fase fcc - bct. Se procede por otro mecanismo, es decir de nucleación de las dislocaciones 
parciales o de Shockley y del deslizamiento progresivo a lo largo de los planos adyacentes. Después de la reorientación la sección transversal cuadrada de los nanocables cambia a una forma rómbica. La diferencia en los mecanismos de reorientación proviene de la diferencia en la falla de la energía de apilamiento inestable y de la dependencia de la orientación de la densidad electrónica.

La transformación de fase en nanocables de estructura cristalina bcc fue estudiada y observada por (Sandoval \& Urbassek, 2009) han tratado de aclarar si en la superficie inducida de pseudo - elasticidad pueden ocurrir en nanocables bcc de $\mathrm{Fe}$, con otras direcciones de eje usando simulación de DM, observando bajo diferentes tipos de tensiones de tracción (Sandoval \& Urbassek, 2009) que a medida que sube la temperatura hay un claro cambio brusco en la longitud del nanocable correspondiente a la estructura bcc se observa la transición de fases fcc / hcp. La tensión de tracción aplicada enérgicamente mejorará la transformación de fase de bcc a fcc / hcp pero impide el proceso inverso. La reorientación inducida por la superficie de estructuras hexagonal compactas (hcp) en nanocables de $\mathrm{Zr}$ ha sido demostrado por (Li et al., 2010) utilizando la simulación con DM (Li et al., 2010). Cabe señalar que el mecanismo de la reorientación en nanocables hcp no es a través de la nucleación y propagación de límites de maclas como es en los cristales de los nanocables fcc (Liang \& Zhou, 2005) y bcc (Li et al., 2010), (Cao, 2010) el cambio de una reorientación o transformación de fase en nanocables hcp se da a través de una transición de fase de dos pasos bcc- hcp. En resumen la reorientación inducida por la superficie y la pseudo-elasticidad en nanocables metálicos de estructura hcp suelen ser de un comportamiento producto de la transformación de fases.

\section{RESULTADOS}

En el siguiente estudio de la transformación de fases en el nanocable de $\mathrm{Cu}$ producto de la orientación y velocidad durante la deformación por tracción, se inicío con la creación del nanocable (NC) metálicos de $\mathrm{Cu}$, de estructura cristalina macroscópica del tipo FCC. Se han preparado NCs con diferentes morfologías y secciones transversales cuadrada de lado $L_{x^{\prime}} L_{y}$, circular con radio r y hexagonal con lado del hexágono $l$ como se ilustra en la figura siguiente, la longitud de todos los nanocables (NCs) con los siguientes tamaños: 4a x 4a x 30a, 6a x 6a x 30a, 8a x 8a x 30a, donde ' $a$ ' es el parámetro de la red cristalina del metal. El tamaño real de los NCs depende de la forma como se corta a partir de un cristal con estructura FCC, es decir de la combinación de la sección transversal y la orientación cristalográfica del eje axial del nanocable, además del parámetro de red ' $a$ '; de igual manera depende el número total de átomos. Corresponde $a_{c u}=0.361 \mathrm{~nm}$ para cobre. La deformación por tensión unitaria del NC se ha estudiado en dos direcciones cristalográficas: $\langle 001\rangle$ y $\langle 111\rangle$ Para el caso de la orientación $\langle 001\rangle$ los ejes cartesianos $\mathrm{x}, \mathrm{y}, \mathrm{z}$ están orientados a lo largo de $\langle 100\rangle,\langle 010\rangle,\langle 001\rangle$; mientras que para la orientación $\langle 111\rangle:\langle 1 \overline{1} 0\rangle,\langle 11 \overline{2}\rangle,\langle 111\rangle$ (Ver figura 1).

La simulación del nano cable de $\mathrm{Cu}$ se realiza con condiciones de frontera periódica en el eje $z$, es decir el eje axial del $\mathrm{NC}$, mientras que en los ejes $\mathrm{x}$, y no se aplica ninguna condición, es decir las superficies laterales son libres. Antes de aplicar la tensión, primero el NC es relajado mediante el método de gradiente conjugado con la finalidad de obtener el mínimo local de la energía. Luego el NC es equilibrado a la temperatura deseada de $100 \mathrm{~K}$ durante
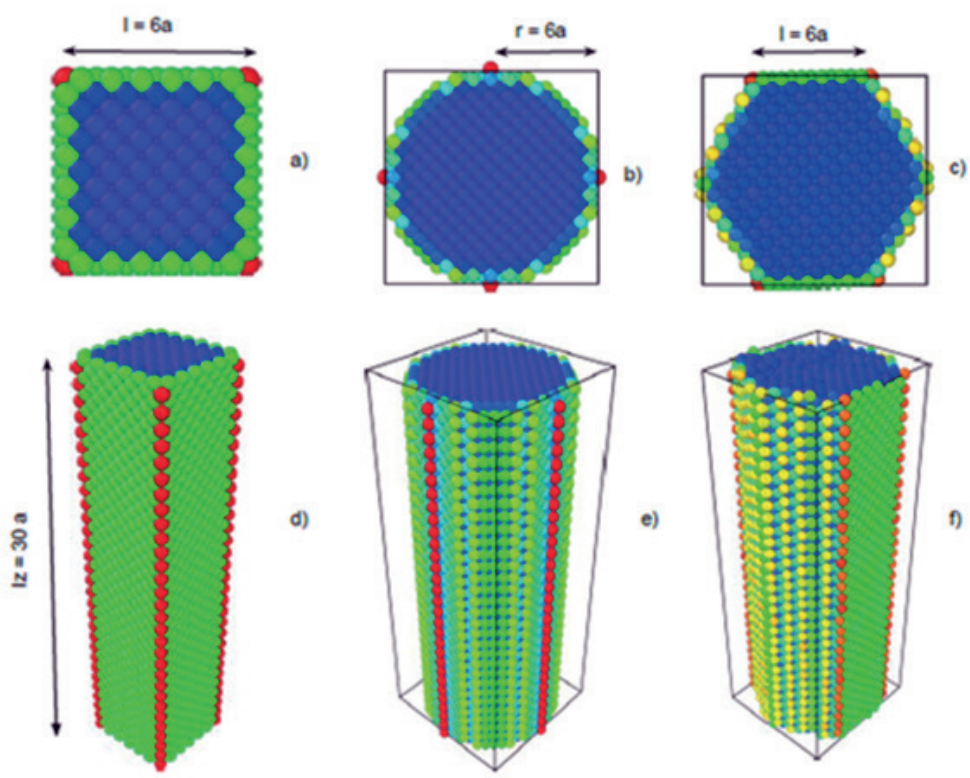

Figura 1. Esquemas de las diferentes morfologías y secciones transversales de los NCs para el caso particular de dimensiones $6 a \times 6 a \times 30 a$. A) sección cuadrada, b) sección circular, c) sección hexagonal, en d), e) y f) se muestran las diferentes morfologías de las superficies laterales. Los átomos están coloreados de acuerdo a la energía potencial. 
20000 pasos de integración o 40 ps. Durante el proceso de equilibrio, la temperatura se mantiene constante usando el termostato de (Nosé, 1984) de tal manera que al final del proceso de equilibración la longitud del $\mathrm{NC}$ adquiere su valor inicial $L_{z 0}$ luego de la equilibración al NC se aplica una tensión uniaxial con la siguiente velocidad $1 \times 10^{9} \mathrm{~s}^{-1}$ (Ver figura 2, tabla 1).

De la observación de las gráficas tensión-deformación aplicadas a los nanocables de cobre de diferentes dimensiones deformados a la misma velocidad y en la misma orientación, las diferencias en el módulo de elasticidad es mínima la diferencia con respecto al área de la sección transversal del cable y siempre el de menor área obtiene el más alto valor especialmente en las de la forma circular y cuadrada y cayendo este valor para la forma hexagonal con respecto a la orientación casi es homogénea la reacción en los tres nanocables, la presencia de la región plástica se observa como si fuera un serrucho con una ondulación moderada y una tendencia a ser prolongada. De acuerdo a las diferentes morfologías de los NCs de las figura 2, graficadas sus curvas tensión - deformación dependen de los siguientes factores; la estructura cristalina, composición y métodos de obtención. La modificación de la morfología
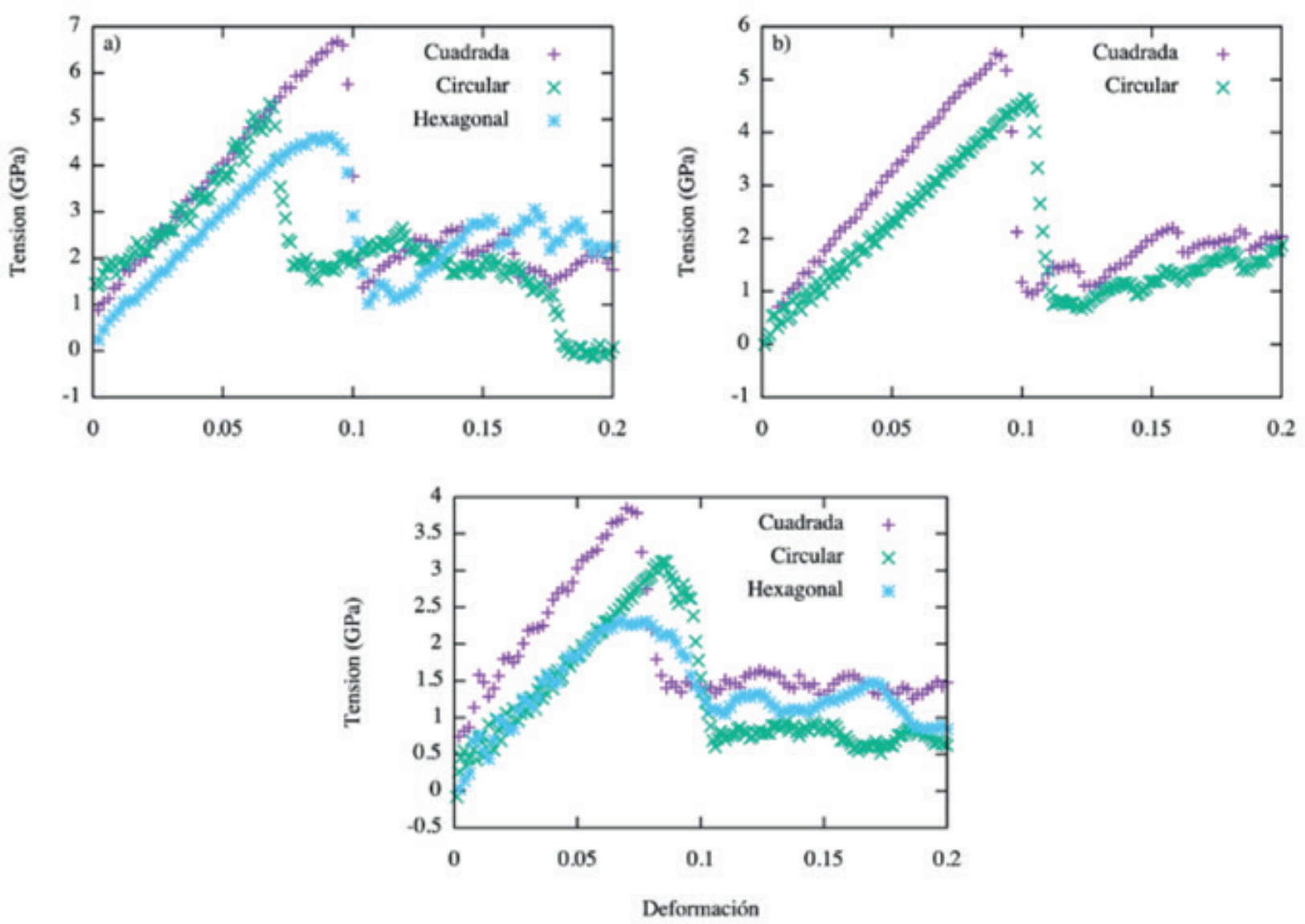

Figura 2. Curvas tensión-deformación de los NCs de a) - Cu, b) - Ag y c) Au con secciones transversales en forma cuadrada, circular y hexagonal. Las secciones transversales de los NCs tiene las siguientes dimensiones; lado $=6 x$ a para la cuadrada, radio $=6 x a$ para la circular y $\mathrm{I}=8 \mathrm{xa}$ para la hexagonal

Tabla 1. Valores del módulo de elasticidad $(E)$ y del límite de elasticidad $(\mathrm{Tm})$ de las curvas tensión deformación referidas a la figura 2(a) del NC de cobre, correspondientes a diferentes formas de la sección transversal y el lado de sus dimensiones, las cuales son deformadas en dirección $<001>$ y con velocidad de deformación igual a $1 \times 10^{9} \mathrm{~s}^{-1}$

\begin{tabular}{cccc}
\hline Forma sección transversal & Tamaño & $\mathbf{T}_{\mathbf{m}}(\mathbf{G P a})$ & $\mathbf{E}(\mathbf{G P a})$ \\
& 4 & 5.3 & 53 \\
circular & 6 & 8.0 & 77 \\
& 8 & 5.8 & 55 \\
cuadrada & 4 & 5.0 & 36 \\
& 6 & 8.0 & 60 \\
hexagonal & 8 & 7.0 & 55 \\
& 4 & 5.0 & 55 \\
\hline
\end{tabular}


es una manera de controlar las características funcionales de los nanomateriales. Por lo tanto la diferencia principal se relaciona con la geometría de la sección transversal y morfología de la sección lateral. A su vez la morfología de la superficie lateral en gran medida es determina por el eje axial y el sistema de planos cristalográficos. En general las propiedades de los cristales (monocristales) son anisotrópicos es decir dependen de la orientación cristalográfica. Los nanocables analizados en el presente trabajo constan de un solo cristalito y en consecuencia en ellas debe manifestarse la anisotropía de las propiedades mecánicas (Ver figura 3 y tabla 2).

Las dos graficas correspondientes a los nanocables de cobre de sección transversal cuadrada sometidas a la misma velocidad de deformación y diferente dirección a la temperatura de $100 \mathrm{~K}$, con respecto a la distribución de fases, sobre la base del análisis del vecino común CNA la cual nos muestra la evolución de transformación de fases que se desarrolla de acuerdo a la curva tensión-deformación:
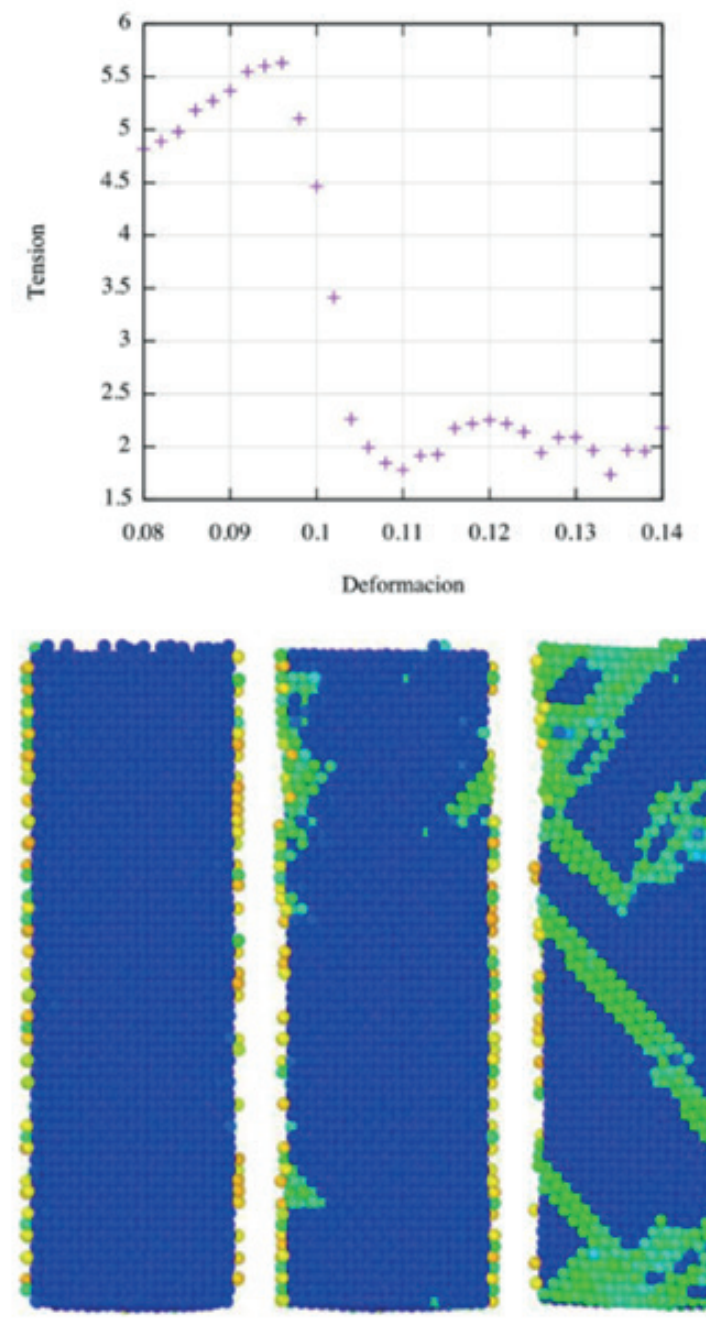

0.098

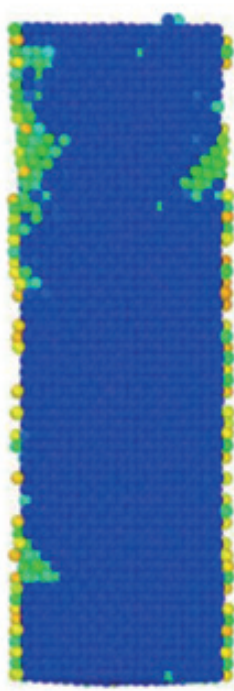

$e=0.100$

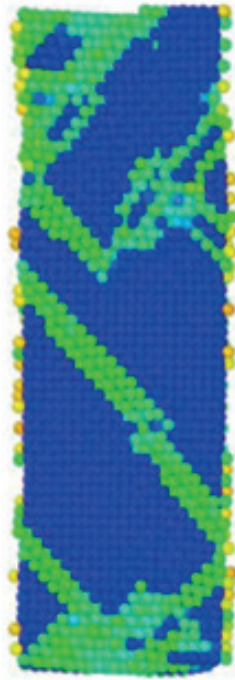

$e=0.102$

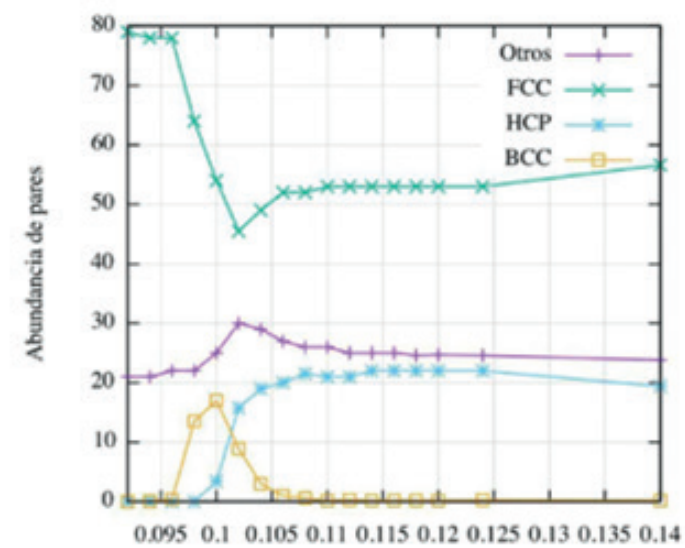

Deformacion
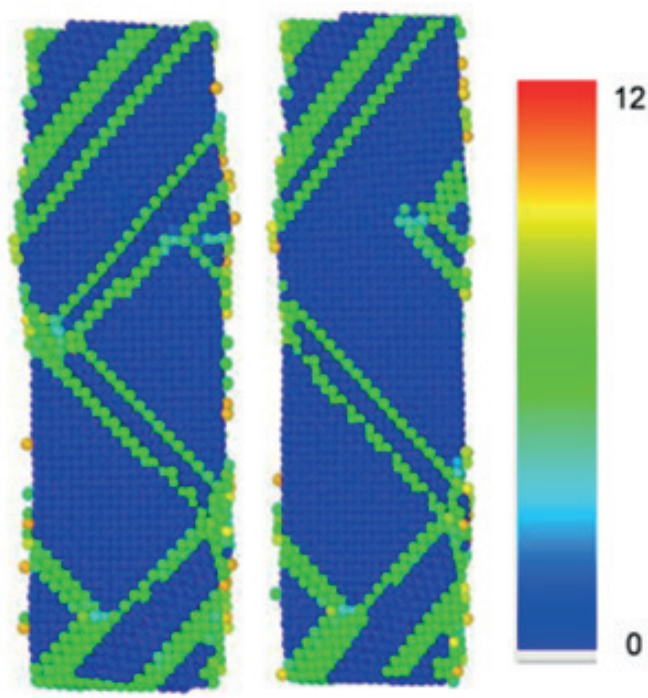

CSP

Figura 3. Deformación de los NCs de sección cuadrada con lado $6 x a$ a temperatura $100 \mathrm{~K}$. a) Curva tensión - deformación, b) Variación de la abundancia de pares, c) Configuraciones atómicas

Tabla 2. Presenta el límite de elasticidad y el módulo de elasticidad del nanocable de cobre y corresponde a la figura 3

\begin{tabular}{cccc}
\hline Forma y tamaño & velocidad & orientación & $\mathrm{Cu}_{m}(G P a) E(G P a)$ \\
\hline \multirow{2}{*}{ cuadrado 8a } & $1 \times 10^{9} \mathrm{~s}^{-1}$ & {$[001]$} & 6.6558 \\
& $1 \times 10^{9} \mathrm{~s}^{-1}$ & {$[111]$} & 9.0100 \\
\hline
\end{tabular}


la fase de transformación presenta un máximo pico del $70 \%$ y luego cae y se muestra constante en un $65 \%$ la diferencia puede decirse que se encuentra en la superficie, con relación a la fase HCp presenta una transformación del $10 \%$ en forma constante y a la fase BCC la transformación es mínima llegando a presentar menos del 1\%.

Los resultados con respecto a la curva tensióndeformación nos presentan una transformación de fases y no una reorientación de la red y podemos decir que mejores resultados nos muestra el nanocable deformado con la velocidad de $1 \times 10^{9} \mathrm{~s}^{-1}$ y en la dirección [111] con valores de Módulo de Elasticidad de $100 \mathrm{GPa}$ y como límite de Elasticidad de $9 \mathrm{GPa}$. La configuración atómica nos muestra los diferentes estados del nanocable deformado producto de la transformación de fases a través de una mezcla de dominios de FCC y HCp preferentemente con una simetría aceptable.

\section{RESULTADOS}

De las dos orientaciones, el mayor módulo de elasticidad es de $\mathrm{E}=100 \mathrm{GPa}$ y límite de fluencia es $\mathrm{Tm}=9.0 \mathrm{GPa}$ posee el nanocable orientado en la dirección $\langle 111\rangle$ de sección transversal cuadrada, es decir tiene la mayor resistencia aunque pierde en deformación crítica promedio $\left(\epsilon_{\mathrm{c}}=0.08\right)$, luego se hace más frágil, además el nanocable con esta orientación presenta una región con pseudoelasticidad bien definida. Los mecanismos de deformación y transformaciones estructurales en los nanocables, con la aplicación del esfuerzo o tensión en la región elástica produce el desplazamiento coordinado de todos los átomos en dirección del esfuerzo aplicado dando como resultado el estiramiento de los enlaces atómicos acumulados como la energía elástica.

En cambio la deformación plástica se produce bajo la acción de esfuerzos de corte (tangenciales) mediante el deslizamiento de las partes individuales del cristal en ciertos planos cristalográficos llamados planos de deslizamiento a lo largo de las direcciones de deslizamiento formado un sistema de deslizamiento.

En las figuras podemos observar la respuesta mecánica a la tensión de tracción aplicada en los nanocables de $\mathrm{Cu}$ en la dirección [001] a la temperatura de $100^{\circ} \mathrm{K}$. las configuraciones atómicas instantáneas están coloreadas de acuerdo al valor del parámetro de centro de simetría (PCS). Los átomos superficiales para los cuales el PCS es mayor que 12 , no se muestran para facilitar la visualización. Los átomos de color azul son aquellos que tienen la estructura libre de defectos; los átomos verdes representan las fallas de apilamiento y aparecen en las direcciones de color rojo. Como se puede apreciar cuando la deformación es, inmediatamente después de lo que corresponde al límite de fluencia, la deformación plástica se inicia con la emisión de dislocaciones parciales y formación de fallas de apilamiento de las aristas del nanocable.

La técnica de análisis de pares comunes, permite identificar la presencia de estructuras locales como FCC, HCP, BCC y otros. Como se pueden observar en las figuras se grafica la variación o abundancia de las estructuras locales, mediante otros se define a la cantidad de átomos que forman estructuras locales diferentes a FCC, HCP y $\mathrm{BCC}$, principalmente son los que se encuentran en la superficie. En primer lugar hay una correlación directa entre la curva tensión-deformación, y el porcentaje de la abundancia de pares. En la región elástica casi el en un rango de $60 \%$ a $80 \%$ de átomos se encuentran formando estructuras FCC, unos $10 \%$ a $20 \%$ la estructura $\mathrm{HCp}$, la formación de la estructura $\mathrm{BCC}$ es mínima y el porcentaje de otras formaciones están en un rango de 20\% a 40\%. Al inicio de la zona elástica la cantidad de estructuras FCC disminuye fuertemente hasta la mitad de su porcentaje inicial y simultáneamente aumenta la abundancia de las estructuras BCC hasta un mínimo porcentaje y luego tiende a mantener esta constante. Lo cual nos dice que ocurre una transformación parcial de FCC $\rightarrow$ BCC. A partir de una deformación cercada a la caída del límite de elasticidad esta nueva fase BCC se transforma en HCp. A deformaciones superiores a 106 las estructuras BCC desaparecen totalmente, mientras que las FCC y HCP alcanzan cierto valor y se mantiene casi constante. Cuando la estructura FCC alcanza el mínimo valor a la deformación 0.102 las otras estructuras alcanzan el valor máximo de $40 \%$, esto debido a que aumenta la superficie por los planos adicionales que salen a la superficie.

\section{REFERENCIAS}

Amigo, N., Gutiérrez, G., \& Ignat, M. (2014). Atomistic simulation of single crystal copper nanowires under tensile stress: Influence of silver impurities in the emission of dislocations. Computational Materials Science, 87, 76-82. https://doi.org/10.1016/j.commatsci.2014.02.014

Cammarata, R. C. (1994). Surface and interface stress effects in thin films. Progress in Surface Science, 46(1), 1-38. https:// doi.org/10.1016/0079-6816(94)90005-1

Cao, A. (2010). Shape memory effects and pseudoelasticity in bcc metallic nanowires. Journal of Applied Physics, 108(11), 113531. https://doi.org/10.1063/1.3506413

Chen, D.-L., \& Chen, T.-C. (2005). Mechanical properties of Au nanowires under uniaxial tension with high strain-rate by molecular dynamics. Nanotechnology, 16(12), 2972-2981. https://doi.org/10.1088/0957-4484/16/12/041

Delogu, F. (2010). Numerical Investigation of the Cubic-toTetragonal Phase Transition in Ag Nanorods. The Journal of Physical Chemistry C, 114(8), 3364-3370. https://doi. org/10.1021/jp9091199

Diao, J., Gall, K., \& Dunn, M. L. (2003). Surface-stress-induced phase transformation in metal nanowires. Nature Materials, 2(10), 656-660. https://doi.org/10.1038/nmat977

Diao, J., Gall, K., \& Dunn, M. L. (2004). Surface stress driven reorientation of gold nanowires. Physical Review B, 70(7), 075413. https://doi.org/10.1103/PhysRevB.70.075413

Emundts, A., Nowicki, M., \& Bonzel, H. P. (2002). Experimental absolute step and kink formation energies on $\mathrm{Pb}(111)$ vicinal surfaces. Surface Science, 496(1), L35-L42. https://doi. org/10.1016/S0039-6028(01)01614-4 
Gall, K., Diao, J., Dunn, M. L., Haftel, M., Bernstein, N., \& Mehl, M. J. (2005). Tetragonal Phase Transformation in Gold Nanowires. Journal of Engineering Materials and Technology, 127(4), 417-422. https://doi. org/10.1115/1.1924558

Gumbsch, P., \& Daw, M. S. (1991). Interface stresses and their effects on the elastic moduli of metallic multilayers. Physical Review B, 44(8), 3934-3938. https://doi.org/10.1103/ PhysRevB.44.3934

Haftel, M. I. (1993). Surface reconstruction of platinum and gold and the embedded-atom model. Physical Review B, 48(4), 2611-2622. https://doi.org/10.1103/PhysRevB.48.2611

Ibach, H. (1997). The role of surface stress in reconstruction, epitaxial growth and stabilization of mesoscopic structures. Surface Science Reports, 29(5), 195-263. https://doi. org/10.1016/S0167-5729(97)00010-1

Jung, K., Cho, M., \& Zhou, M. (2012). Thermal and mechanical response of [0001]-oriented GaN nanowires during tensile loading and unloading. Journal of Applied Physics, 112(8), 083522. https://doi.org/10.1063/1.4759282

Kollár, J. (2003). Calculation of surface stress for fcc transition metals. Physical Review B, 68(24). https://doi.org/10.1103/ PhysRevB.68.245417

Kulkarni, A. J., Zhou, M., Sarasamak, K., \& Limpijumnong, S. (2006). Novel Phase Transformation in ZnO Nanowires under Tensile Loading. Physical Review Letters, 97(10), 105502. https://doi.org/10.1103/PhysRevLett.97.105502

Li, S., Ding, X., Li, J., Ren, X., Sun, J., Ma, E., \& Lookman, T. (2010). Inverse martensitic transformation in $\mathrm{Zr}$ nanowires. Physical Review B, 81(24), 245433. https://doi.org/10.1103/ PhysRevB.81.245433

Liang, W., \&Zhou,M.(2005).Pseudoelasticity of SingleCrystalline $\mathrm{Cu}$ Nanowires Through Reversible Lattice Reorientations. Journal of Engineering Materials and Technology, 127(4), 423-433. https://doi.org/10.1115/1.1928915

Ma, F., Ma, S.-L., Xu, K.-W., \& Chu, P. K. (2008). Interfaceinduced pseudoelastic behavior in Bi-metal multilayer nanowires. Applied Physics Letters, 92(12), 123103. https:// doi.org/10.1063/1.2902293
Ma, F., \& Xu, K. (2006). Surface induced electron redistribution: A mechanism for mechanical strengthening of $\mathrm{Au}$ nanowires. Scripta Materialia, 55(10), 951-954. https://doi. org/10.1016/j.scriptamat.2006.07.002

Ma, F., Zhang, J.-M., \& Xu, K.-W. (2005). Surface-energydriven abnormal grain growth in $\mathrm{Cu}$ and $\mathrm{Ag}$ films. Applied Surface Science, 242(1), 55-61. https://doi.org/10.1016/j. apsusc.2004.07.068

Nosé, S. (1984). A molecular dynamics method for simulations in the canonical ensemble. Molecular Physics, 52(2), 255-268. https://doi.org/10.1080/00268978400101201

Park, H. S., \& Zimmerman, J. A. (2005). Modeling inelasticity and failure in gold nanowires. Physical Review B, 72(5), 054106. https://doi.org/10.1103/PhysRevB.72.054106

Saitoh, K., \& Liu, W. K. (2009). Molecular dynamics study of surface effect on martensitic cubic-to-tetragonal transformation in Ni-Al alloy. Computational Materials Science, 46(2), 531-544. https://doi.org/10.1016/j. commatsci.2009.04.025

Sandoval, L., \& Urbassek, H. M. (2009). Finite-Size Effects in Fe-Nanowire Solid-Solid Phase Transitions: A Molecular Dynamics Approach. Nano Letters, 9(6), 2290-2294. https:// doi.org/10.1021/n19004767

Sutrakar, V. K., \& Mahapatra, D. R. (2008). Coupled effect of size, strain rate, and temperature on the shape memory of a pentagonal $\mathrm{Cu}$ nanowire. Nanotechnology, 20(4), 045701. https://doi.org/10.1088/0957-4484/20/4/045701

Sutrakar, V. K., \& Roy Mahapatra, D. (2011). Size and temperature dependent stability and phase transformation in single-crystal zirconium nanowire. Journal of Nanoparticle Research, 13(10), 5335. https://doi.org/10.1007/s11051011-0519-4

Wan, J., Fan, Y. L., Gong, D. W., Shen, S. G., \& Fan, X. Q. (1999). Surface relaxation and stress of fcc metals: $\mathrm{Cu}, \mathrm{Ag}, \mathrm{Au}, \mathrm{Ni}$, $\mathrm{Pd}, \mathrm{Pt}, \mathrm{Al}$ and $\mathrm{Pb}$. Modelling and Simulation in Materials Science and Engineering, 7(2), 189-206. https://doi. org/10.1088/0965-0393/7/2/005 
